\title{
Study on detection and recognition of phase contamination and multimode propagation in ionosphere for OTHR
}

\author{
Li Xue ${ }^{1,2 a)}$, Deng Wei Bo ${ }^{1}$, and Jiao Pei Nan ${ }^{2}$ \\ ${ }^{1}$ Harbin Institute of Technology. \\ No.92 Xidazhi Street, Nangang District, Harbin, Heilongjiang, 150001, China \\ ${ }^{2}$ China Research Institute of Radiowave Propagation. \\ No.36 East Xianshan Road, Chengyang District, Qingdao, Shandong, 266107, \\ China
}

a)lixue1201@126.com

\begin{abstract}
Recognition of ionosphere phase contamination and multimode propagation is an important step in rejection of clutter spectrum spread for OTHR. Based on the algorithm of time reversibility, this paper proposes a method based on eigenvalue decomposition to detect multimode propagation. Besides, a cascaded technique composed of the two methods above is proposed to recognise contaminations. Another detection algorithm based on time series Hankel matrix is proposed, which will be suitable for more widely application. For the first time, the method based on backscatter sounding is also proposed for multimode recognition, and a hybrid method based on time reversibility and Hankel matrix is also proposed to distinguish phase contamination and multimode propagation.
\end{abstract}

Keywords: time reversibility, eigenvalue decomposition, Hankel matrix, backscatter sounding, detection and recognition

Classification: Electron devices, circuits, and systems

\section{References}

[1] A. Bourdillon, F. Gauthier, and J. Parent, "Use of maximum entropy spectral analysis to improve ship detection by over-the-horizon radar[J]," Radio Science, vol. 22, no. 2, pp. 313-320, 1987.

[2] J. Parent and A. Bourdillon, "A method to correct HF skywave backscattered signals for ionospheric frequency modulation[J]," IEEE Trans. Antennas Propag., vol. 36, no. 1, pp. 127-135, 1988.

[3] P. E. Howland and D. C. Cooper, "Use of the Wigner-Ville distribution to compensate for ionospheric layer movement in high-frequency sky-wave radar systems[J]," IEEE Proceedings-F, vol. 140, no. 1, pp. 29-36, 1993.

[4] S. J. Anderson and Y. I. Abramovich, "Adaptive ionospheric distortion correction technique for HF skywave radar[J]," Proc. 1996 IEEE National Radar conf., Ann Arbor, May 1996. 
[5] X. guo, "Study on Skywave Over-the-Horizon Radar Signal Processing[M]," A Dissertation Submitted to Nanjing University of Science and Technology for the Degree of Doctor of Philosophy, 2004.

[6] C. L. DiMonte and K. S. Arun, "Tracking the frequencies of superimposed time-varying harmonics[J]," Proc. 1990 IEEE National Radar Conf., pp. 2539-2542, 1990.

\section{Introduction}

Ionosphere phase contamination and multimode propagation arouse OTHR clutter spectrum spread which would influence the performance of low speed target detection and sea state remote sensing. Aim at this problem, a good many researches have been done in recent years.

The popular correction methods of phase contamination include maximum entropy spectral analysis [1], phase gradient algorithm [2], frequencytime analysis [3], and so on. The correction methods of multimode propagation include the conversion from multimode to single-mode [4] and algorithm using circular operation of subtraction for sea clutter echo [5]. If there coexists of phase contamination and multimode propagation effects, all methods above fail. Paper [5] used high-resolution time frequency distribution to detect object directly.

Obviously, the correction methods differ from each other according to phase contamination and multimode propagation existing alone or simultaneously. It will not obtain the expected gain when using these methods incorrectly and what's more, it could deteriorate detection performance.

As mentioned above, to reject spectrum spread effectively, it is necessary to judge and recognize the following four situations:

I) Echo signal with neither phase contamination nor multimode;

II) Echo signal with phase contamination only;

III) Echo signal with multimode effect only;

IV) Echo signal with both phase contamination and multimode.

Few paper has researched on this problem until now. Paper [4] introduced a time reversibility method simply to detect phase contamination, which could not recognize multimode propagation and be invalid for the four cases above.

This paper proposes the method of eigenvalue decomposition to realize multimode propagation detection, and the cascaded using of time reversibility and eigenvalue decomposition succeeds in recognizing case I and III, which can also estimate the number of the propagation modes. For the first time, an algorithm suitbale for case I and III based on time series Hankel matrix is proposed, which consumes less computation time compared with the cascaded technique and dispenses with the hypothesis of contamination function identity. And for the first time a method based on prior ionosphere frequency sweep backscatter sounding is proposed to recognize multimode. A hybrid method based on time reversibility and Hankel matrix is proposed to realize 
the recognition of situation II and IV.

\section{Detection and recognition of phase contamination and mul- timode propagation}

\subsection{Algorithm based on time reversibility for phase contami- nation}

Assume that the uncontaminated echo signals from $\mathrm{K}$ adjacent range cells satisfy i.i.d and share the same contamination function. Echo sequence from the kth cell is expressed as:

$$
x_{k}=D s_{k} ; k=1,2, \ldots, K
$$

where $s_{k}$ is the uncontaminated echo sequence, and $\mathrm{D}$ is a contamination matrix.

$$
\begin{aligned}
& s_{k}^{T}=\left[s_{k}\left(t_{0}\right), s_{k}\left(t_{0}+T_{R}\right), \ldots, s_{k}\left(t_{0}+(N-1) T_{R}\right)\right] \\
& D=\operatorname{diag}[1, \exp (j \varphi(1)), \ldots, \exp (j \varphi(N-1))]
\end{aligned}
$$

$T_{R}$ denotes pulse repetition interval, $\mathrm{N}$ is the number of pulses within a coherent integration period, and $\varphi(n), \mathrm{n}=0,1, \&, \mathrm{~N}-1$ denotes the phase contamination function.

The covariance matrix of the $\mathrm{K}$ adjacent range cells echo is $R=X X^{H} / K$. The time reversibility of $\mathrm{R}$ is expressed by $R=J R^{*} J$, where $\mathrm{J}$ is the permutation matrix. The necessary condition under which echo signal satisfies time reversibility is that the diagonal matrix $\mathrm{D}$ shares the same property with $\mathrm{R}$, viz. $D=J D^{*} J$.

If the ionosphere phase perturbation exists, the diagonal matrix $\mathrm{D}$ will not satisfy time reversibility and neither is the echo signal. That is an evidence to determine whether the echo signal is phase-contaminated. In engineering, we usually determine this through verifying whether the signal subspace of echo covariance matrix is orthogonal with its time-inverse matrix noise subspace.

If there exists only multimode signal without phase perturbation, the uncontaminated echo sequence is:

$$
s_{k}^{T}=s_{k 1}^{T}+s_{k 2}^{T}+\cdots+s_{k r}^{T}
$$

Where $\mathrm{r}$ denotes the number of propagation modes. The covariance matrix is denoted as

$$
R=S S^{H} / K=\sum_{i=1}^{r} \sum_{j=1}^{r} R_{i j}
$$

Exp.4 is the sum of self-covariance and inter-covariance matrix of the propagation modes. Because different mode signals are incoherent, exp.(4) is a zero matrix. And the self-covariance matrix of each mode satisfies time reversibility. So the method can not be used to detect the existence of multimode propagation. 


\subsection{Algorithm based on eigenvalue decomposition for multi- mode}

According to modern spectral analysis theory, the number of information sources is corresponds to the number of echo sequence covariance matrix main eigenvalues.

Consider that uncontaminated sea clutter first-order Bragg peak appears in pair, the number of main eigenvalues is twice as propagation modes. If the received signal is ground clutter, or the signal only contains first-order positive (or negative) sea clutter for the reason of wind direction and velocity, the number of main eigenvalues is equal to propagation modes. According to this characteristic multimode propagation detection can be made.

If the echoes suffered from phase contamination, its time invariance was destroyed. Even no multimode effects exist, the main eigenvalue is still multivalued. Then the method becomes unavailable for multimode detection.

\subsection{Cascaded application of time reversibility and eigenvalue decomposition}

For the cases I and III, if the result of no phase contamination can be made using time reversibility method firstly, then the multimode effect can be detected using eigenvalue decomposition algorithm in addition. Fig. 1a)-d) denote emulational sea clutter spectrum according to the cases of I-IV above, where is added by $-30 \mathrm{~dB}$ noise. A denotes mean value of inner product between signal vector and noise vector, and $\mathrm{K}=0.01$ denotes the orthogonal

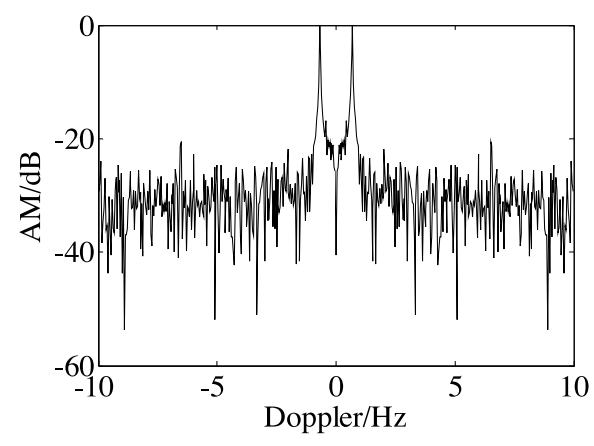

a) Original spectrum

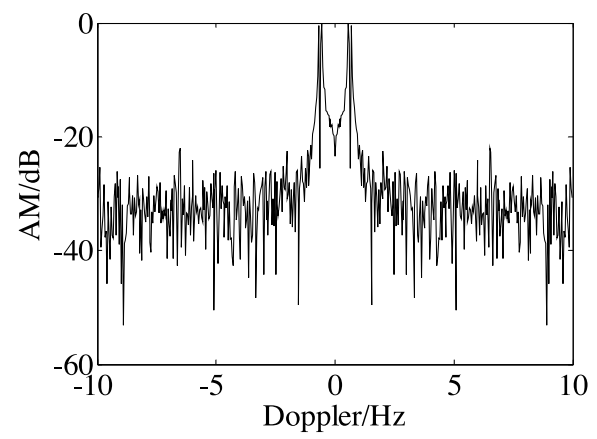

c) Spectrum added multimode effect

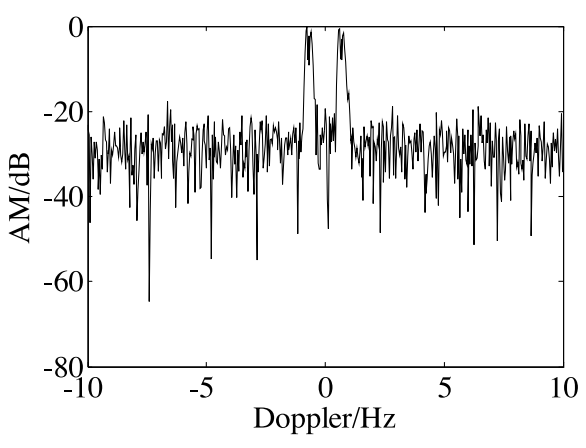

b) Spectrum added phase contamination

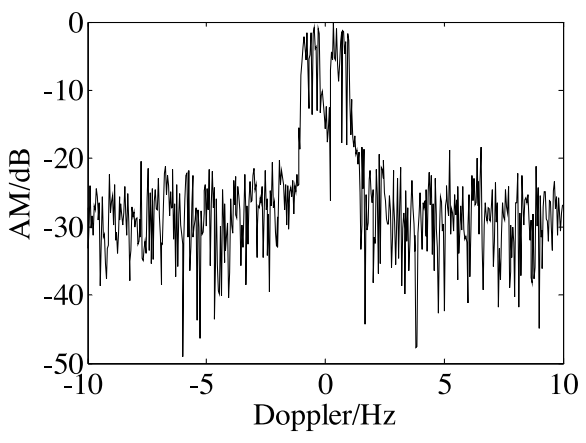

d) Spectrum with phase and multimode contamination

Fig. 1. Emulational sea clutter spectrum 
threshold; $\mathrm{M}$ and $\mathrm{N}$ denoted the number of main eigenvalues and the number of propagation modes, respectively.

The results applied the cascaded method of time reversibility and eigenvalue decomposition to sea clutter spectrum in Fig. 1a)-b) are as follow:

1) For case $\mathrm{I}$ : $\mathrm{A}=4.1 \times 10-5<\mathrm{K}, \mathrm{M}=2, \mathrm{~N}=1$, so there is neither phase contamination nor multimode;

2) For case II: $A=0.23>K$, so there is phase contamination, but the multimode is unknown;

3) For case III: $A=4.6 \times 10-5<\mathrm{K}, \mathrm{M}=4, \mathrm{~N}=2$, so there is no phase contamination but multimode, and the mode number is 2 ;

4) For case IV: $A=0.34>K$, so there is phase contamination, but the multimode is unknown.

Obviously, the cascaded algorithm can recognize case I and III, but fail to recognize case II and IV.

\subsection{Algorithm based on Hankel matrix}

Assume signal $s(n)$ is composed of $\mathrm{r}$ time-variant sinusoidal components:

$$
s(n)=A_{1} \cos \left(\phi_{1}(n)\right)+\cdots+A_{r} \cos \left(\phi_{r}(n)\right)
$$

The instantaneous frequency of each component is expressed as:

$$
\omega_{i}(n)=\phi_{i}(n)-\phi_{i}(n-1) \quad i=1, \ldots \ldots, r
$$

Hankel matrix $\mathrm{H}$ constructed by $s(n)$ is as follows:

$$
H=\left[\begin{array}{llll}
s(1) & s(2) & \cdots & s(c) \\
s(2) & s(3) & \cdots & s(c+1) \\
\vdots & \vdots & \vdots & \vdots \\
s(N-c+1) & s(N-c) & \cdots & s(\mathrm{~N})
\end{array}\right]
$$

Where $c=3 r$ denotes the number of columns.

Paper [6] presented several properties of matrix $\mathrm{H}$ :

Property 1: The rank of $\mathrm{H}$ is $\mathrm{r}$ if the complex sinusoid frequency is a constant.

Property 2: $\mathrm{H}$ is a full rank matrix if the complex sinusoid frequency is time-variant.

Obviously, exp. (4) can be used to denote the echo signal for OTHR, where $\mathrm{r}$ indicates the number of sea clutter first-order peaks. If ionosphere phase contamination exists in the echo signal, the frequency is time-variant and the Hankel matrix is a full rank matrix. If the contamination does not exist, the frequency is a constant and the rank of the Hankel matrix is r. Consider that the sea clutter first-order peeks appear in pair, the propagation mode number is $\mathrm{r} / 2$.

Using this method to detect the spectrum in fig. $1 \mathrm{a})$-d), $R$ denotes the rank of $\mathrm{H}$ and column number is $\mathrm{c}=12$. The results are as follow:

1) For case $I$ : $R=2, N=1$, so there is neither phase contamination nor multimode; 
2) For case III: $\mathrm{R}=4, \mathrm{~N}=2$, so there is no phase contamination but multimode, and the mode number is 2 ;

3) For case II and IV: R=12, so there is phase contamination, but multimode is unknown.

Hankel matrix method has the same performance with cascaded method that invalid in case II and IV, but it does not need restricted conditions as cascaded method. Just as paper [6] indicated that the $\mathrm{H}$ matrix method is enslaved to noise, so the noise must be suppressed firstly before using this method. This paper succeeds in using SVD (singular value decomposition) to suppress noise.

\subsection{Multimode propagation recognition based on backscat- tering detection}

Backscatter sounding is an important technique of ionosphere surveillance. It has the same propagation path as OTHR. A simulation example of backscatter ionogram is shown in Fig. 2 d) where the signal region surrounded by green lines is multi-mode and the other three signal regions are single mode. The recognition of received signals with operation frequency in a range cell can be realized on the basis of the propagation modes information extracted from the backscatter ionogram.

Situation II and IV can be dealt with using this cascaded method of backscattering and time reversibility (or Hankel matrix).

Compared with eigenvalue decomposition and Hankel matrix methods, the backscattering method introduces new information resource, and can detect multimode while phase contamination existed. However, it fails in the mode number estimation.

\section{Experimental tests}

HF backscatter fixed frequency sounding shares the same echo characteristics with OTHR. So the algorithms above can be tested using the data collected from the backscatter equipment. A set of fixed-frequency experimental data spectrum of 12th May, 2009 is shown in Fig. 2 a). The operation frequency is $8.532 \mathrm{MHz}$, group distance is $650 \mathrm{~km}$, and CIT is $12.8 \mathrm{~s}$. Two methods of cascaded algorithm and Hankel matrix are applied to the data separately. Here, the column of Hankel matrix is 6 . Results are given as follow:

1) The cascaded algorithm: $A=8.4 \times 10-4<K, M=2, N=2$, so there is no phase contamination but multimode;

2) Hankel matrix algorithm: $\mathrm{R}=2, \mathrm{~N}=2$, so there is no phase contamination but multimode.

The sea clutter power density spectrum of backscatter fixed frequency sounding on 17th May, 2009 is shown in Fig. 2 b). Parameters of operation frequency, group distance and CIT are $10.077 \mathrm{MHz}, 1150 \mathrm{~km}, 102.4 \mathrm{~s}$, respectively. Fig. 2 c) is the backscatter ionogram in adjacent time. Fig. 2 d) is the ionogram after propagation mode extraction, where the blue "+" indicates the corresponding position of operation frequency and group distance. 


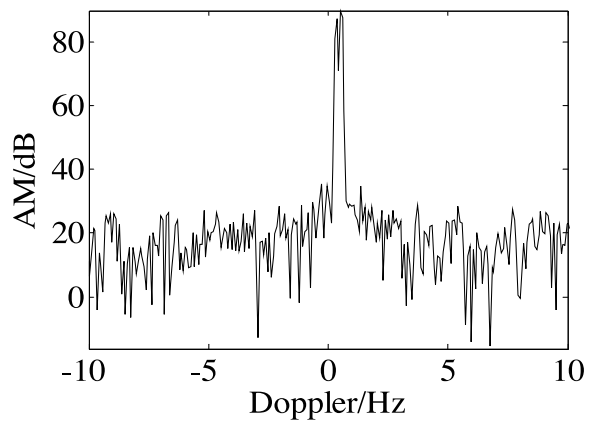

a) Multimode spectrum of backscattering at 13:40:00, 12th May, 2009

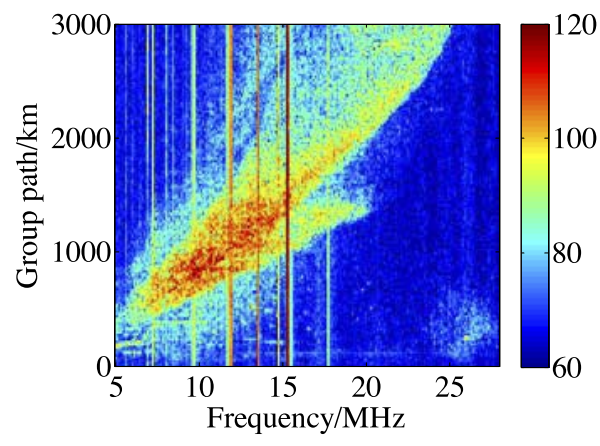

c) Backscattering sweep ionogram at 15:30:00, 17th May, 2009

Fig. 2. Backscattering test pattern

Table I. Phase contamination detection for the backscattering experimental data

\begin{tabular}{ccc}
\hline Methods & Judgement basis & Conclusion \\
\hline Time reversibility & $\begin{array}{c}\mathrm{A}=0.39>\mathrm{K} \\
\text { Nonorthogonal }\end{array}$ & Phase contamination \\
\hline Hankel matrix & Matrix rank is 12 & Phase contamination \\
\hline Backscattering detecting & Multimode extraction & Multimode effect \\
\hline
\end{tabular}

Using time reversibility and Hankel matrix to detect phase contamination, let the rank of $\mathrm{H}$ matrix is 12. The result is in table I. Because of the existence of phase contamination, backscatter sounding method can only be applied to recognize multimode propagation effect. It is apparent to see the multimode in Fig. $2 \mathrm{~d}$ ) according to the attracted borderline and region.

Conclusion can be made that the experimental data suffered from multimode propagation and phase contamination simultaneously.

\section{Conclusion}

Hankel matrix or the cascaded algorithm (time reversibility and eigenvalue decomposition) can realize the recognition for case I and III of echo signal and estimate the mode number. But they can not detect multimode effect. The method based on backscatter sounding succeeds in multimode detection un- 
der the condition of phase contamination coexists. The united application of the proposed methods above can recognize case I-IV. These decontamination methods can be chosen to provide for the consequent signal processing.

\section{Acknowledgments}

This work was supported by the National Nature Science Foundation of China (NSFC) under grant 61040002. 